\title{
Comparisons of Efficacy and Safety between Triple (Inhaled Corticosteroid/Long-Acting Muscarinic Antagonist/Long-Acting Beta-Agonist) Therapies in Chronic Obstructive Pulmonary Disease: Systematic Review and Bayesian Network Meta-Analysis
}

\author{
Hyun Woo Lee $^{a}$ Hyung Jun Kim ${ }^{b}$ Eun Jin Jang ${ }^{c}$ Chang-Hoon Lee ${ }^{b}$ \\ aDivision of Pulmonary and Critical Care Medicine, Department of Internal Medicine, Seoul Metropolitan \\ Government-Seoul National University Boramae Medical Center, Dongjak-gu, Seoul, Republic of Korea; 'bivision \\ of Pulmonary and Critical Care Medicine, Department of Internal Medicine, Seoul National University Hospital, \\ Jongno-Gu, Seoul, Republic of Korea; 'Department of Information Statistics, Andong National University, Andong, \\ Republic of Korea
}

\section{Keywords}

Pulmonary disease $\cdot$ Chronic obstructive $\cdot$ Respiratory therapy - Symptom flare-up - Mortality · Drug-related side effects and adverse reactions

\footnotetext{
Abstract

Background: Various combinations of inhaled corticosteroid (ICS), long-acting muscarinic antagonist (LAMA), and long-acting beta-agonist (LABA) have been used as triple therapy for stable chronic obstructive pulmonary disease (COPD). Objective: Our study was conducted to answer whether there were significant differences among various combinations in efficacy, for reducing exacerbation or mortality, and in safety, for increasing cardiovascular events or pneumonia. Method: We searched parallel-group randomized controlled trials (RCTs) comparing ICS/LAMA/LABA with other inhaled drugs in patients with stable COPD for at least 12 weeks in PubMed, EMBASE, the Cochrane Library, and clinical trial registries from inception to December 31, 2019. We conducted a network meta-analysis with Bayesian statistics
}

using a random-effects model with heterogeneous variance structure (PROSPERO, CRD42019126757). Results: Nine different combinations of ICS/LAMA/LABA were identified in 21 RCTs containing 29,892 patients with moderate to very severe COPD. We could not find any significant evidence suggesting a better treatment for reducing total exacerbations or allcause mortality among ICS/LAMA/LABA combinations. There were also no significant differences in moderate to severe exacerbation, COPD-related mortality, or cardiovascular diseaserelated mortality among ICS/LAMA/LABA combinations, and the risk of major adverse cardiovascular events was not different. A significantly lower risk of pneumonia was found in fluticasone propionate (FP)/glycopyrrolate/salmeterol (SAL) than FP/tiotropium/SAL \{median odds ratio [OR] (95\% credible interval $[\mathrm{Crl}])=0[0-0.72]\}$ and $\mathrm{FP} /$ umeclidinium/SAL \{median OR $(95 \% \mathrm{Crl})=0$ [0-0.97]\}. Conclusion: There were no significant differences in clinical outcomes, including acute exacerbation and all-cause mortality among various ICS/LAMA/LABA combinations in patients with moderate to very severe COPD.

(c) 2021 The Author(s)

Published by S. Karger AG, Basel karger@karger.com www.karger.com/res

Karger $\stackrel{\text { ' }}{5}$

BOPEN ACCESS
(C) 2021 The Author(s)

Published by S. Karger AG, Basel

This is an Open Access article licensed under the Creative Commons Attribution-NonCommercial-4.0 International License (CC BY-NC) (http://www.karger.com/Services/OpenAccessLicense), applicable to the online version of the article only. Usage and distribution for commercial purposes requires written permission. 


\section{Introduction}

Chronic obstructive pulmonary disease (COPD) is a global public health problem owing to its high morbidity, mortality, and economic or social burden [1-3]. For the treatment of patients with stable COPD, long-acting beta-agonists (LABAs), long-acting muscarinic antagonists (LAMAs), and inhaled corticosteroids (ICSs) have been used for decades. These inhaled drugs are beneficial for reducing respiratory symptoms, decreasing the frequency and severity of acute exacerbations, improving quality of life, and ameliorating exercise intolerance [4]. In particular, combination therapies, such as ICS/LABA [5] or LAMA/LABA [6], have been shown to be superior to monotherapy. Additive effects of ICS on LABA have been explained by several pharmacological mechanisms such as increasing density or decreasing desensitization of beta-adrenoceptors [7]. Potential benefits of a combination of 2 bronchodilators, LAMA and LABA, have been understood by their complementary mechanisms [8]. However, there is still a proportion of patients with COPD who suffer from acute exacerbation or dyspnea, even during dual combination therapy [9]. In addition, no prospective controlled randomized clinical trials have shown that dual therapy reduced mortality risk in patients with COPD $[5,10]$. Recent studies have increasingly focused on triple therapy or on combinations of ICS/LAMA/ LABA $[4,11]$.

ICSs/LAMAs/LABAs had significant benefits for the reduction of acute exacerbations [9, 12-15], symptoms $[15,16]$, and rescue medication use [17], and the improvement of lung function $[13,15,16]$ compared with ICSs/ LABAs or LAMAs/LABAs in randomized controlled trials (RCTs). Furthermore, we previously showed that triple therapy reduced all-cause mortality compared with other dual or single treatments [18]. Currently, ICSs/LAMAs/ LABAs are recommended for some patients with persistent breathlessness or who experienced exacerbations, despite the dual therapies [4]. Various single-inhaler ICS/ LABA/LAMA fixed-dose combinations are currently available or under development [11]. Although each ICS/LAMA/LABA has been expected to yield similar benefits and adverse events, this belief has not been based on sufficient scientific evidence. There have been only a few head-to-head RCTs conducted [13, 19], and only one network meta-analysis (NMA), which is outdated [20].

The present systematic review (SR) and Bayesian NMA aimed to compare treatment outcomes, including acute exacerbation, all-cause mortality, and adverse events, between different ICS/LAMA/LABA combinations in patients with stable COPD by including all eligible RCTs.

\section{Materials and Methods}

\section{Protocol and Registration}

Our study protocol was drafted following the Preferred Reporting Items for Systematic Review and Meta-Analysis (PRISMA) extension statement for the reporting of SRs incorporating NMAs on healthcare interventions [21] and the Bayeswatch guidelines for reporting our results using Bayesian statistics [22]. The study protocol was approved and uploaded on the international prospective register of SRs (CRD42019126757, PROSPERO).

\section{Eligibility Criteria}

Studies were considered eligible when the following inclusion criteria were met: (1) patients with stable COPD, (2) inhaled administration of ICS/LAMA/LABA combinations, (3) parallel-design RCTs that clearly reported acute exacerbation or mortality as a prespecified study outcome, (4) treatment duration of $\geq 12$ weeks, and (5) clinical studies published in English.

\section{Primary Outcome}

The co-primary outcomes were total exacerbation and allcause mortality. The secondary outcomes were moderate to severe exacerbation, COPD-related mortality, cardiovascular disease-related mortality, and safety profiles, including the incidence of major adverse cardiovascular events (MACE) and pneumonia.

\section{Data Sources and Searches}

We used the Peer Review of Electronic Search Strategies checklist [23] to develop a search strategy and to identify the relevant literature in PubMed, Embase, and the Cochrane Library (search date: December 31, 2019). The search terms included controlled vocabulary and free text. In brief, our search strategy comprised "COPD" AND inhaled drugs ("ICS" AND "LAMA" AND “LABA") AND randomized controlled design. Individual inhaled drugs in each drug class were also searched.

In addition, we searched websites of the EU Clinical Trial Register, the US National Library of Medicine, and registers of pharmaceutical companies, including AstraZeneca, Boehringer Ingelheim, GlaxoSmithKline, and Novartis. To obtain more information on unpublished data, we contacted the corresponding author or the person in charge on the study. Manual searches were performed with the references described in previous SRs. The detailed method of the search strategy is described in Appendix S1 and PROSPERO.

\section{Study Selection}

The process of study selection was performed by independent 2 authors (H.W.L and H.J.K.) in accordance with the PRISMA flow diagram [24]. To improve interobserver reliability, calibration exercises were performed with a random sample of 200 articles (agreement $=98.0 \%$, Cohen's $k=0.66$ [substantial agreement]). The 2 reviewers independently identified duplicated articles, screened potentially eligible articles using the titles and abstracts, and reviewed the full text to confirm whether the 
screened studies were in agreement with our eligibility criteria. In cases of disagreement, we referred to the original articles and discussed them together.

\section{Data Extraction and Quality Assessment}

We discussed the data collection process and structured a pilot data extraction format to evaluate study quality and the synthesis of outcome variables. This data extraction sheet was pilot-tested on 5 randomly selected included studies and refined accordingly. The data extraction process was performed by 2 independent reviewers (H.W.L. and H.J.K.). The collected data items were as follows: (1) study information (e.g., authors, publication, year of study, study identifier, study duration, eligibility criteria, the number of included patients for intention-to-treat analysis, and pre-established study outcomes); (2) baseline characteristics of the study patients (e.g., age, sex, smoking status, and ethnicity); (3) clinical information of the study patients (e.g., mean postbronchodilator forced expiratory volume in the first second, COPD severity (Global Initiative for Chronic Obstructive Lung Disease [GOLD] stage), history of acute exacerbation within 1 year and symptom scores; and (4) study outcomes (e.g., number of patients with total or moderate to severe exacerbations of COPD, the number of all-cause mortality cases and their causes of death, the number of patients with MACE, and the number of patients with pneumonia until the last follow-up). In cases in which the number of patients was not presented, we digitized the raw data from the Kaplan-Meier curve containing the information on the time to the first event [25]. The severity of COPD exacerbation was determined by either the Exacerbations of Chronic Pulmonary Disease Tool [26] or use of a healthcare resource [27]. Mild exacerbation was defined as a condition with worsening symptoms resolved by short-acting bronchodilators. Moderate exacerbation was defined as a condition with worsening symptoms that requires systemic corticosteroids or antibiotics. Severe exacerbation was defined as a condition with worsening symptoms that requires hospitalization or emergency room visit. Any controversial issue regarding the data extraction process was resolved by discussion.

\section{Network Geometry for the Included Studies}

The network geometry of the included studies was evaluated to clarify which triple inhaled therapies were included for the comparisons, how many direct comparisons were conducted, how many patients were assigned to each treatment, and whether a treatment or comparison was preferred or avoided. In the geometry of the network, each individual inhaled drug and their combination therapies were expressed as nodes, and a head-to-head comparison between 2 different treatments was expressed as an edge between nodes. The number of head-to-head comparisons was expressed as the thickness of the edges and written in the middle of the edges.

Risk of Bias Assessment within and across Individual Studies

The risk of bias (ROB) of the included studies was independently assessed at the study level by 2 reviewers (H.W.L. and H.J.K.) using the Cochrane ROB tool [28]. We investigated the publication bias using funnel plots and Egger's test if the direct comparisons included 3 or more RCTs. Any further controversy related to ROB assessment was discussed with the third author (C.H.L.).

Clinical Outcomes of ICS/LAMA/LABA
Data Synthesis and Analysis

Assuming different variance of the odds ratio (OR) for efficacy and safety in different ICSs/LAMAs/LABAs, we optimized a random-effects model using a heterogeneous variance structure [29, 30]. We used noninformative prior distributions that were assumed to have a normal or uniform distribution [31].

The probability of being the best treatment among individual ICSs/LAMAs/LABAs was estimated and ranked based on the methodology of the surface under the cumulative ranking curve [32]. The median ORs with 95\% credible intervals (CrIs) and the posterior probability of the OR exceeding $1(p(\mathrm{OR}>1))$ were derived from the estimated posterior distributions to find significant superiority between ICSs/LAMAs/LABAs. We considered a $p(\mathrm{OR}$ $>1$ ) of less than 0.025 or more than 0.975 to have a statistical significance.

To identify whether a specific subpopulation in COPD patients has different effectiveness for reducing total exacerbations among the triple therapies, sensitivity and subgroup analyses were planned according to exacerbation history and study duration.

We checked the homogeneity and similarity assumptions by reviewing the baseline characteristics of the included RCTs and the included patients. We assessed the consistency assumption, which implies that the direct estimate is consistent with indirect estimates in a NMA, by using the node-splitting method [33]. We appraised the heterogeneity across studies based on the posterior median value of the standard deviation between studies. Detailed information regarding the data synthesis and analysis is provided in Appendix S2.

\section{Results}

\section{Study Selection and Network Geometry}

We identified total 2,367 records from databases and other sources (Fig. 1). After the removal of duplicates, 1,437 records were screened to find 45 potentially relevant articles or abstracts. We performed a full-text review, and 21 articles met the eligibility criteria of the present study. Excluded references were described with the main reason in Appendix S3. The network geometries characterizing interconnected relationship among inhaled drugs were graphically illustrated for total exacerbation (A) and all-cause mortality (B), which were almost identical (Fig. 2). Direct head-to-head comparisons between ICSs/LAMAs/LABAs were found in 3 studies $[13,19,34]$.

\section{Study and Patient Characteristics}

The characteristics of the included studies and the features of their participants are summarized in Table 1. Twenty RCTs were published between 2007 and 2018, and 1 RCT (TRISTAR study) was not published. In total, 29,892 patients were included. The mean age was approximately 64.6 years, approximately $71.0 \%$ of patients were male, and approximately $40.4 \%$ were current smokers. White or Caucasian and Asian patients were 


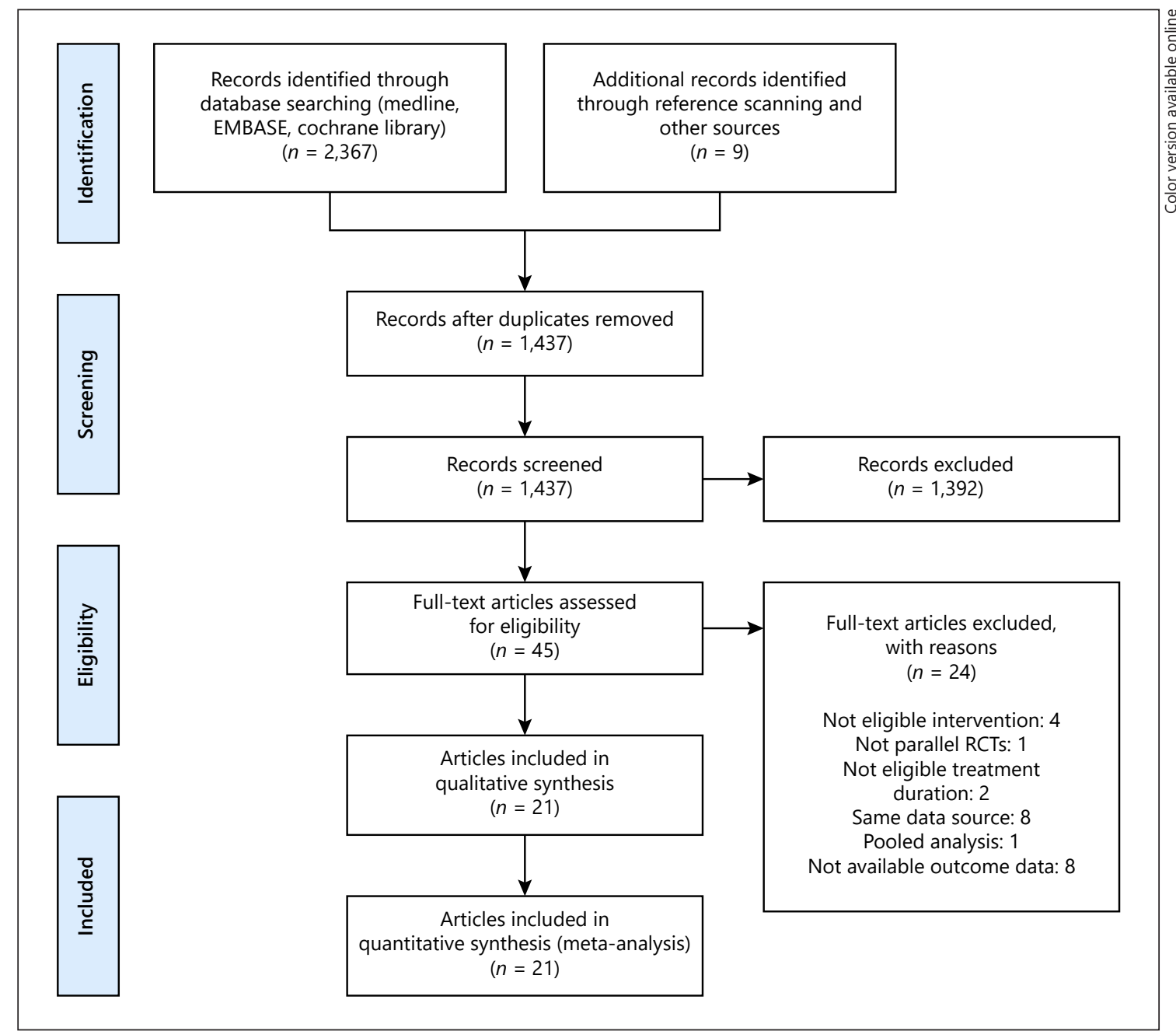

Fig. 1. PRISMA flowchart of study selection for the NMA. NMA, network meta-analysis; PRISMA, Preferred Reporting Items for Systematic Review and Meta-Analysis; RCT, randomized controlled trial.

dominantly enrolled in 9 RCTs of 20,258 patients; other RCTs did not describe ethnicity. Mean post-bronchodilator forced expiratory volume in the first second percentage was approximately $42.2 \%$, and most of the patients had severe COPD; 9 RCTs evaluated 20,477 patients with GOLD grades 3-4, 7 RCTs evaluated 5,172 patients with GOLD grades $2-4$, and 5 RCTs evaluated 4,243 patients with GOLD grades $2-3$. Patients with COPD GOLD grade 1 were not included in our metaanalysis. The percentage of the included patients with at least 1 exacerbation in the previous year was approximately $89.3 \%$, and 9 RCTs included 20,836 patients who had experienced 1 or more exacerbations in the previous year. We found that 23,607 patients with more symptoms (COPD Assessment Test [CAT] $\geq 10$ or modified Medical Research Council $[\mathrm{mMRC}] \geq 2$ ) were enrolled in 12 RCTs.
With regard to interventions, 9 combinations of ICS/ LAMA/LABA were identified: 5,888 patients from 4 RCTs were the fluticasone furoate (FF)/umeclidinium (UME)/ vilanterol (VIL) arm, 3,105 patients from 4 RCTs were the beclomethasone dipropionate (BDP)/glycopyrrolate (GLY)/formoterol (FOR) arm, 2,598 patients from 7 RCTs were the fluticasone propionate (FP)/tiotropium dry powder inhaler (TIO)/salmeterol (SAL) arm, 814 patients from 2 RCTs were the FP/UME/SAL arm, 706 patients from 3 RCTs were the budesonide (BUD)/TIO/FOR arm, 639 patients from 1 RCT were the BUD/GLY/FOR arm, 579 patients from 1 RCT were the FF/TIO/VIL arm, 537 patients from $1 \mathrm{RCT}$ were the $\mathrm{BDP} / \mathrm{TIO} / \mathrm{FOR}$ arm, and 257 patients from 1 RCT were the FP/GLY/SAL arm. The study period was 12 weeks in 8 RCTs, 24 or 26 weeks in 7 RCTs, and 52 weeks in 6 RCTs. With regard to inhaler devices, a dry powder inhaler was most frequently used 


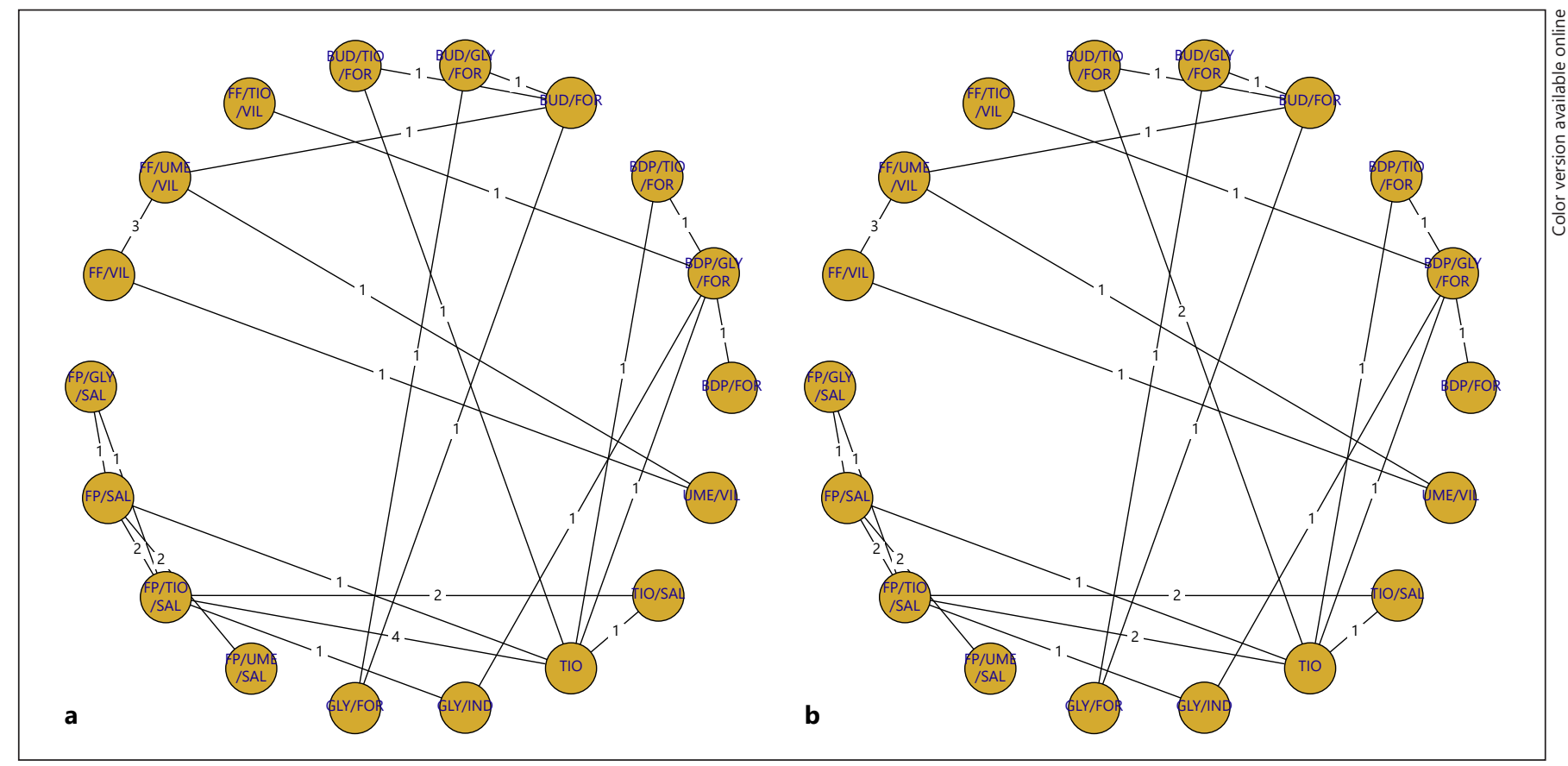

Fig. 2. Network geometry of the direct comparisons in the 21 eligible RCTs; the risk of total exacerbations (a); the risk of all-cause mortality (b). Network expressed individual drugs or their combinations as each node, and a direct comparison of 2 different treatments in an RCT was shown as a line between nodes. The number of direct comparisons was expressed as a number in the middle of

(twenty RCTs) and a metered dose inhaler was also used (6 RCTs). Extrafine particle inhaled therapies were used in 4 RCTs $[12,13,15,34]$.

\section{Assessment of ROB within and across Studies}

By evaluation of ROB within studies, we made an assessment that the included RCTs generally had good study quality and were acceptable for NMA. The details of the ROB assessment are described in Appendix S4. In the exploration of the potential for ROB across studies, neither significant publication bias nor selective reporting was detected (Appendix S5).

\section{Acute Exacerbation}

To compare the risk of total exacerbation among individual ICS/LAMA/LABA combinations, 29,667 patients in 20 RCTs were analyzed. However, we did not find a significant difference between the ICS/LAMA/ LABA combinations for the reduction of total exacerbations (Table 2). In the analysis to compare the efficacy for reducing moderate to severe exacerbations, 23,188 patients in 8 RCTs were analyzed. The combination therapies such as $\mathrm{BUD} / \mathrm{TIO} / \mathrm{FOR}, \mathrm{FP} / \mathrm{UME} /$ a line between nodes. BDP, beclomethasone; BUD, budesonide; FF, fluticasone furoate; FOR, formoterol; FP, fluticasone propionate; GLY, glycopyrrolate; IND, indacaterol; SAL, salmeterol; TIO, tiotropium dry powder inhaler; UME, umeclidinium; VIL, vilanterol; $\mathrm{RCT}$, randomized controlled trial.

SAL, FF/TIO/VIL, and FP/GLY/SAL were not evaluated because of the limited data available. We found no significant difference among the ICS/LAMA/LABA combinations for the reduction of moderate to severe exacerbations. In sensitivity analysis with RCTs including patients with at least 1 exacerbation event in the past year, we found no significant difference in reducing acute exacerbation among available 6 combinations of ICS/LAMA/LABA (Appendix S6). In subgroup analysis according to study duration of $<52$ or $\geq 52$ weeks, there was no significant difference among the triple therapies (Appendix S6).

\section{Mortality}

We analyzed 29,530 patients in 19 RCTs to compare the risk reduction of all-cause mortality between the ICS/LAMA/LABA combinations. No specific ICS/ LAMA/LABA was superior to other ICS/LAMA/LABA combinations for the reduction of all-cause mortality risk (Table 2). Based on the cause of death, 2 NMAs were conducted: one for COPD-related mortality with 16,136 patients in 8 RCTs and the other for cardiovascular disease-related mortality with 18,621 patients in 9 RCTs. 


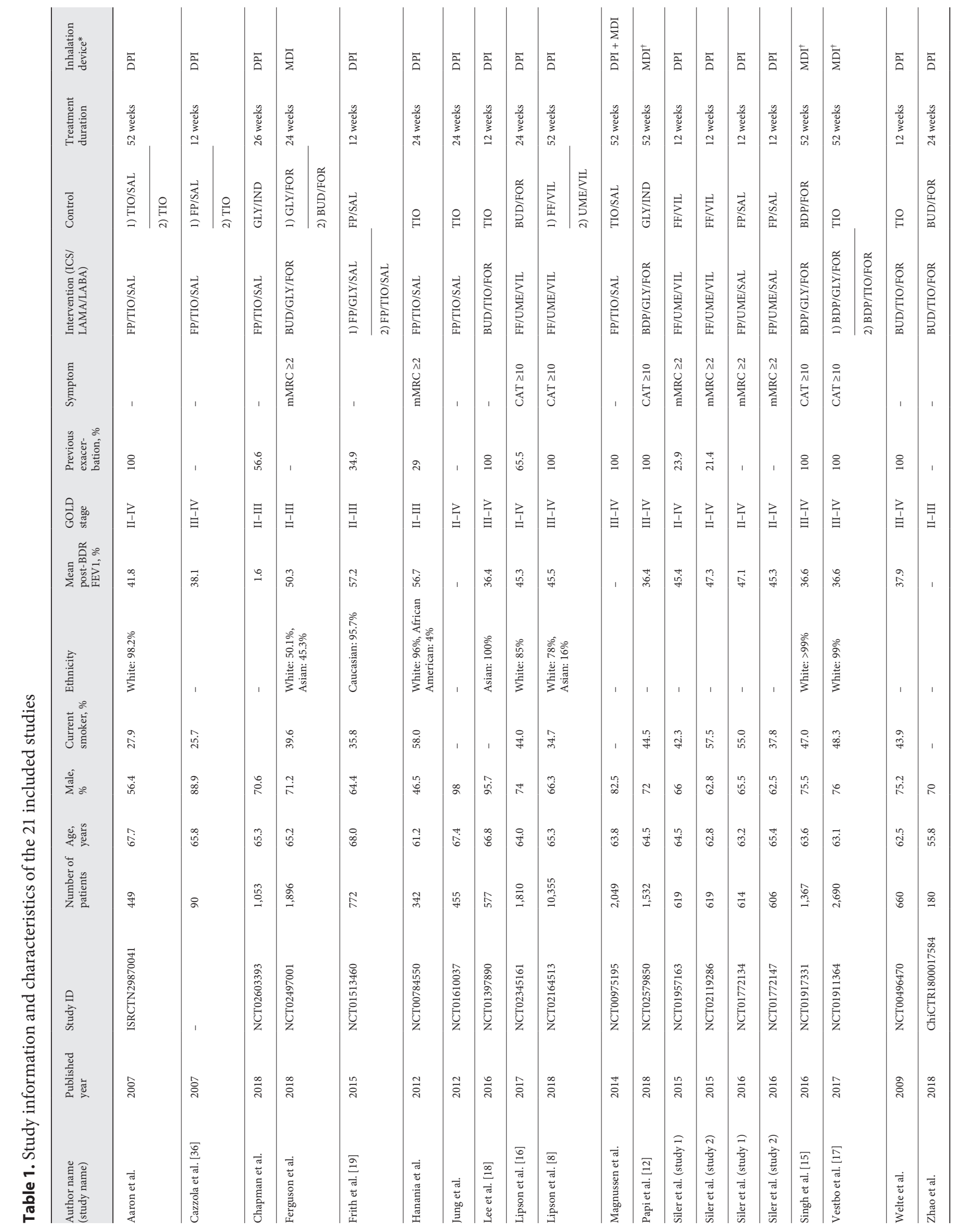




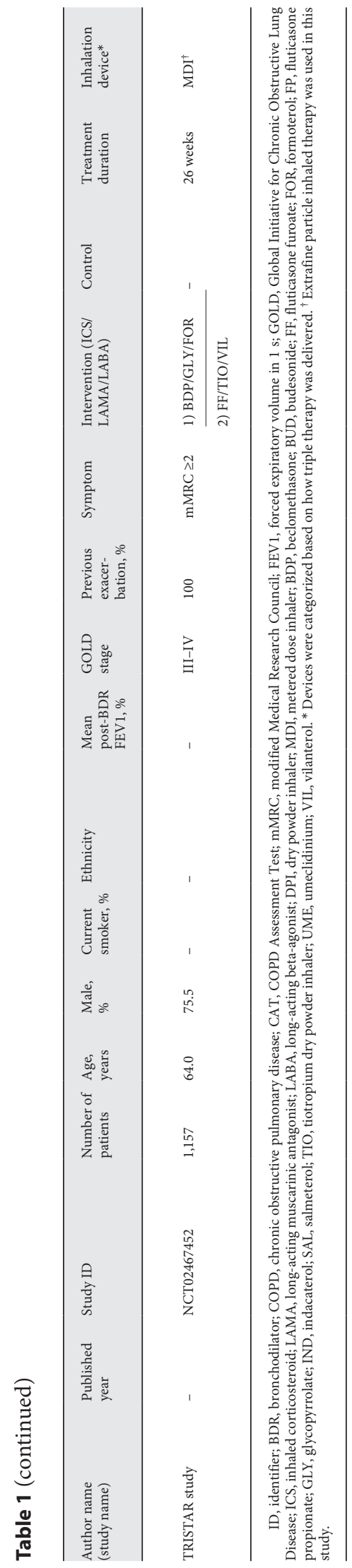

Clinical Outcomes of ICS/LAMA/LABA
Owing to the limited data available, BDP/GLY/FOR, $\mathrm{BDP} / \mathrm{TIO} / \mathrm{FOR}$, and FF/TIO/VIL were not analyzed for COPD-related mortality, and BUD/GLY/FOR, FF/TIO/ VIL, FP/GLY/SAL were not analyzed for cardiovascular disease-related mortality. We found no significant results because the $95 \% \mathrm{CrI}$ was too wide to compare the efficacy of each ICS/LAMA/LABA combination (Table 2).

\section{Adverse Events}

We analyzed 11,736 patients in 8 RCTs to compare the risk of MACE between ICS/LAMA/LABA combinations. FP/UME/SAL and FP/GLY/SAL were not analyzed because of the limited data available. A significant difference was not detected in the comparison of the risk of MACE among ICS/LAMA/LABA combinations because the $95 \% \mathrm{CrI}$ was too wide to compare the safety of each ICS/LAMA/LABA combination (Table 2).

We compared the risk of pneumonia among different ICS/LAMA/LABA combinations in 28,516 patients in 19 RCTs. FP/GLY/SAL was ranked the highest according to surface under the cumulative ranking curve. FP/GLY/SAL showed a significantly lower risk of pneumonia than FP/ TIO/SAL $\{$ median OR $(95 \% \mathrm{CrI})=0[0-0.72]\}$ and $\mathrm{FP} /$ UME/SAL $\{$ median OR (95\% CrI) $=0[0-0.97]\}$ (Table 2).

\section{Consistency Assumption}

The posterior effect size estimated by Bayesian NMA was assumed to be consistent with the effect size estimated by the paired meta-analysis of direct comparisons (Appendix S7). In the statistical test to evaluate the level of inconsistency, the consistency assumption was satisfied for most of the comparisons.

\section{Discussion/Conclusion}

We systematically reviewed RCTs that evaluated ICS/ LAMA/LABA treatment of $\geq 12$ weeks in patients with stable COPD and moderate to very severe GOLD stage. Our NMA indirectly compared the efficacy and safety of various ICSs/LAMAs/LABAs by using a Bayesian method. There were no differences in the reduction in the risk of acute exacerbation or all-cause mortality among different ICSs/LAMAs/LABAs. Owing to the limited number of patients, sufficient analyses could not be performed to investigate the impact of drugs on the incidence of moderate to severe exacerbations, COPD-related mortality, $\mathrm{MACE}$, and cardiovascular disease-related mortality. We did not find any evidence against the common belief that 


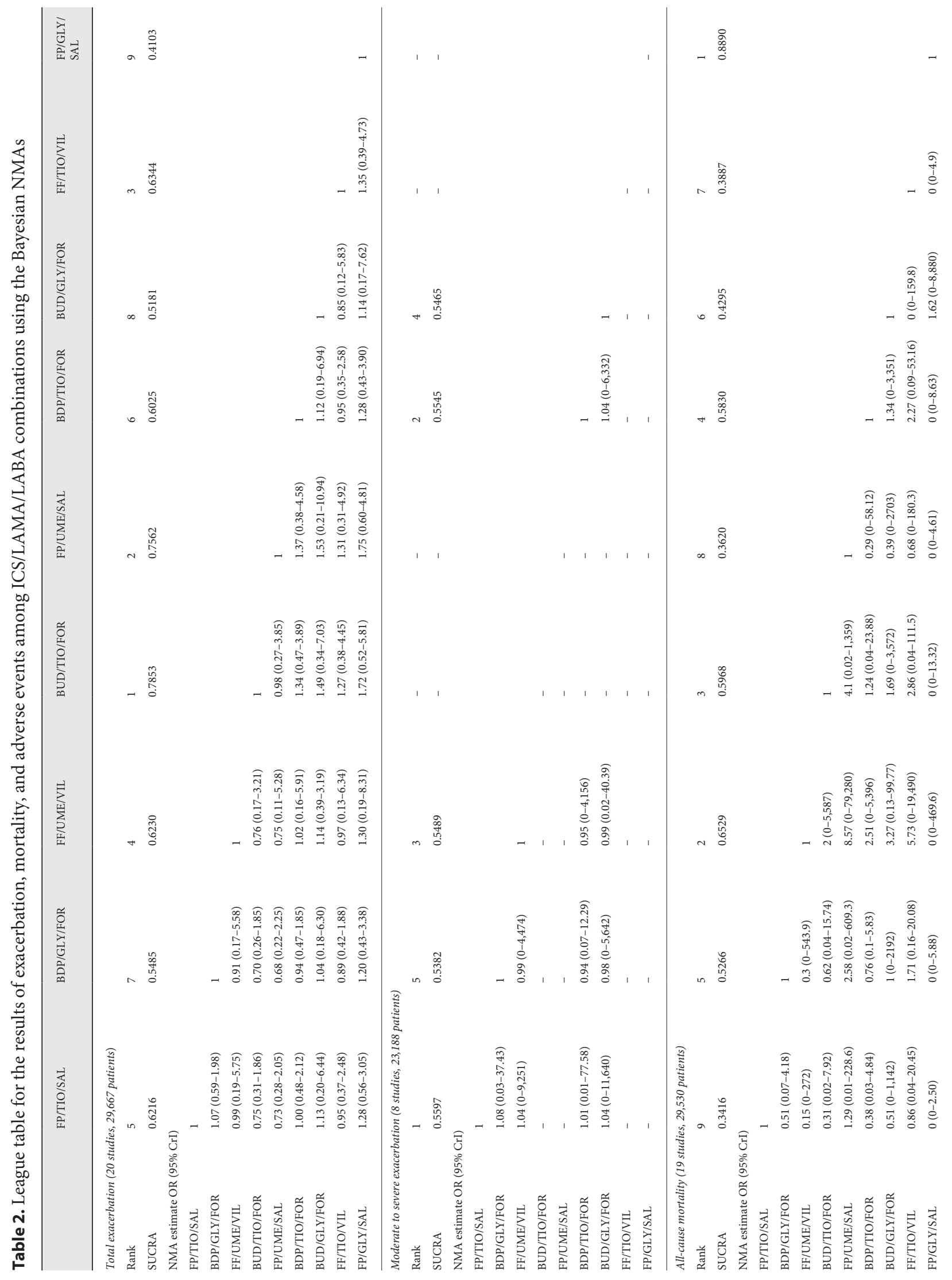




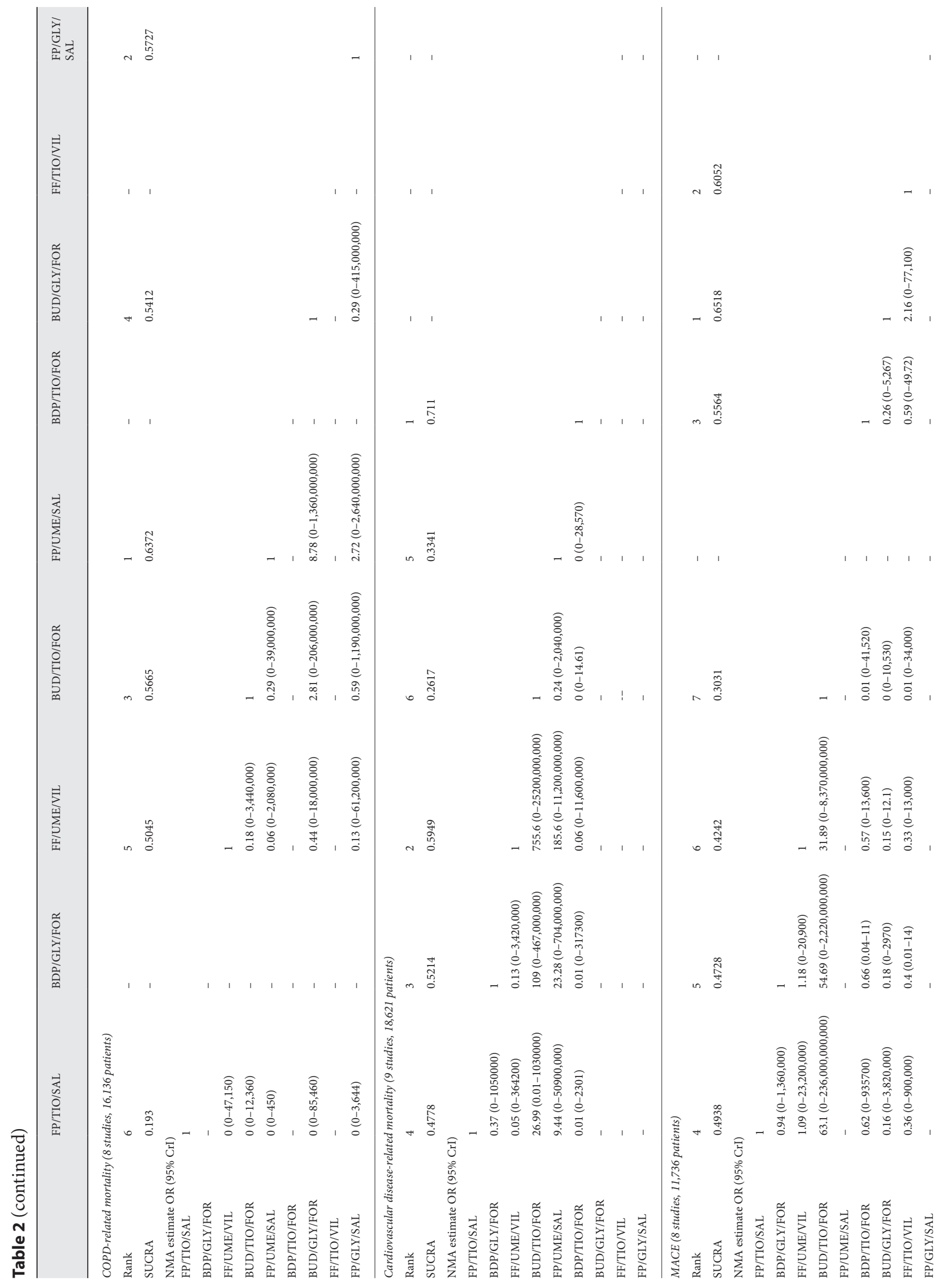




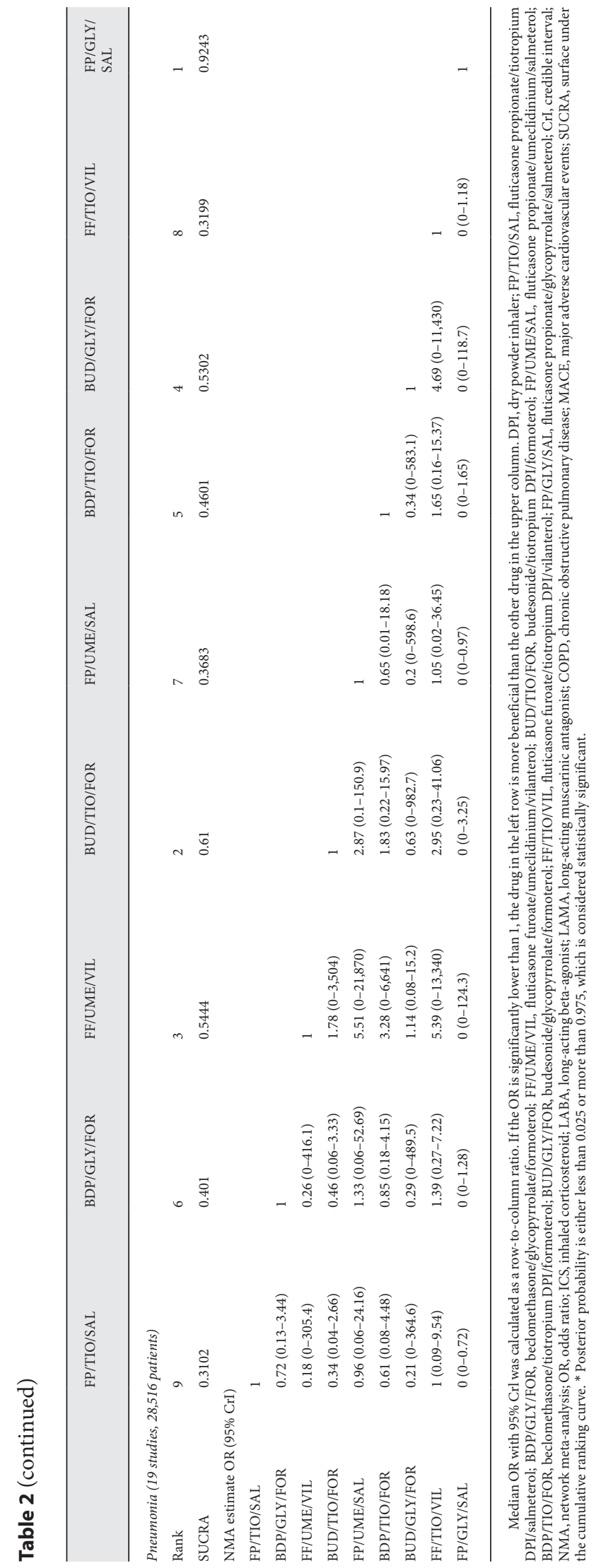

different ICSs/LAMAs/LABAs would have similar efficacy and safety, except that the risk of pneumonia was significantly lower with FP/GLY/SAL than with FP/TIO/ SAL or FP/UME/SAL. Although fluticasone treatment has been reported to be associated with higher risk of pneumonia, FP/GLY/SAL ranked first in lowering pneumonia risk among different ICSs/LAMAs/LABAs.

Recent large RCTs $[9,12-17]$ and meta-analyses [18, 35-38] have shown a superior impact of ICSs/LAMAs/ LABAs in improving lung function, reducing acute exacerbation, and increasing symptoms compared with LAMAs/LABAs or ICSs/LABAs, without an increase in the incidence of adverse events. Although experts expect each ICS/LAMA/LABA to yield similar benefits and adverse events, the supporting scientific evidence is not available. Indeed, in the TRINITY trial, the combination of BDP/ TIO/FOR showed a greater improvement in inspiratory capacity after 12 weeks and had more SGRQ responders at 26 weeks than BDP/GLY/FOR [13]. However, a post hoc analysis revealed that BDP/GLY/FOR resulted in a $29 \%$ greater reduction of acute exacerbations than BDP/ $\mathrm{TIO} / \mathrm{FOR}$ in the subgroup with $>1$ exacerbation in the previous year [39]. In the GLISTEN trial, fewer cardiovascular disease-related adverse events were reported with FP/GLY/SAL than with FP/TIO/SAL [19]. Therefore, we conducted SR with NMA to perform an indirect comparison of the efficacy and safety of individual drug combinations including recently published RCTs $[9,12,14]$.

A previous NMA showed a superior efficacy of BUD/ $\mathrm{TIO} / \mathrm{FOR}$ to $\mathrm{FP} / \mathrm{TIO} / \mathrm{SAL}$ in the reduction of moderate to severe exacerbations $\{\mathrm{OR}(95 \% \mathrm{CI})=0.40[0.21-80]\}[20]$. Although the comparisons between various inhaled drugs for stable COPD were extensively evaluated in the NMA, only 2 combinations of ICS/LAMA/LABA were available, $\mathrm{BUD} / \mathrm{TIO} / \mathrm{FOR}$ and FP/TIO/SAL. This is because the NMA did not include recent RCTs $[9,12,14]$ and could not conduct direct and indirect comparisons between ICSs/ LAMAs/LABAs. In recent years, a wider variety of combinations of ICS/LAMA/LABA have been developed. Indeed, our SR found 9 combinations of ICS/LAMA/LABA compared with other inhaled drugs for the treatment of COPD. Our SR with NMA was the first attempt to compare the efficacy and safety at the level of individual triple therapies that included all available RCTs to date. However, we did not find significant differences in total exacerbation, all-cause mortality, moderate to severe exacerbation, COPD-related mortality, or cardiovascular disease-related mortality, and MACE among different ICS/LAMA/LABA combinations. This supports the expectation that each ICS/LAMA/LABA is equal or similar in benefits and harm. 
Before we started the present study, a thorough literature review was conducted to determine whether the use of a fixed-dose combination (FDC, same as fixed triple therapy) impacted clinical outcomes. An RCT compared the efficacy and safety of FF/UME/VIL between an FDC and a free-dose combination and found no significant differences [40]. The TRINITY trial reported that an FDC lowered the SGRQ scores at 26 and 52 weeks and decreased moderate to severe exacerbation risk in the subgroup with previous exacerbation history [13, 41]. However, it was unclear whether the difference in efficacy was based on the use of the FDC because different drug combinations were compared simultaneously (BDP/GLY/ $\mathrm{FOR}$ as an FDC and BDP/TIO/FOR as a free-dose combination) [13]. A recent pairwise meta-analysis of the drug class reported that the use of FDC or free-dose combination was not significantly related to acute exacerbation, mortality, and safety outcomes [35]. Therefore, it was assumed that FDC therapy would have a trivial impact on clinical outcome.

In our study, pneumonia was observed less frequently in patients treated with $\mathrm{FP} / \mathrm{GLY} / \mathrm{SAL}$ than those treated with FP/TIO/SAL or FP/UME/SAL. A recent NMA showed no difference in pneumonia risk among different LAMAs/ LABAs [42]. We thought that there may be an interaction between ICSs, a known risk factor for the incidence of pneumonia [43], and LAMA, based on several studies [14, 44]. However, we could not identify a plausible mechanism from our analysis to explain the difference in the incidence of pneumonia among those who used the same inhaled drugs, except LAMAs. In addition, because it has been well known that LAMAs do not increase the risk of pneumonia [18], the clinical importance could not be assured.

Our study had several limitations. First, our SR and NMA included RCTs with different patient characteristics, eligibility criteria, and disease severity. Although statistical heterogeneity was found insignificant, qualitative heterogeneity cannot be excluded. Second, more research studies on triple therapies are needed to draw definitive conclusions. The network geometry showed that most of the edges consisted of only one study, which means the result of the only study dominated the result of pooled analysis. In addition, our prespecified outcomes such as moderate to severe exacerbation and mortality more rarely occurred than we expected. In fact, the posterior probability of mortality risk reduction was the considerably higher in FP/GLY/SAL than other ICSs/LAMAs/LABAs, but significant results could not be obtained. In addition, we could not perform various subgroup analyses according to selected phenotypes such as sputum or blood eosinophilia, asthma-COPD overlap syndrome, or frequent exacerbators [45]. Therefore, similar studies are required in stratified COPD patients. Third, most study periods were as short as 1 year or less. There are only 6 RCTs over 52 weeks in our study. At least 1 year of sufficient period of medications is necessary to evaluate the acute exacerbation between interventions [46]. Sensitivity analysis in a previous NMA showed that the risk reduction of acute exacerbation and all-cause mortality by ICSs/LAMAs/LABAs tended to be greater when the study period was longer [18]. Therefore, further studies on ICSs/LAMAs/LABAs with a longer period are needed.

In conclusion, our SR with a Bayesian NMA that included all available relevant RCTs showed no significant differences in acute exacerbation and all-cause mortality among the 9 different ICSs/LAMAs/LABAs used for the treatment of patients with stable COPD. This finding suggested that each ICS/LAMA/LABA has comparable benefits and harms.

\section{Statement of Ethics}

No ethical approval was not required because data from previous published randomized controlled trials in which informed consent was obtained by primary investigators were retrieved and analyzed.

\section{Conflict of Interest Statement}

All authors declare no conflicts of interest associated with the present study. The corresponding author (C.H.L.) affirms that the manuscript is an honest, accurate, and transparent account of the study being reported; that no important aspects of the study have been omitted; and that any discrepancies from the study as planned (and, if relevant registered) have been explained.

\section{Funding Sources}

This study was funded by the SNUH Research Fund (grant no. 23-2017-0020) (website: http://snuh.org/). The funding source had no role in study design, data collection and analysis, decision to publish, or preparation of the manuscript.

\section{Author Contributions}

Study concept and design: H.W.L. and C.H.L. Acquisition of data: H.W.L., H.J.K., and C.H.L. Analysis and interpretation of data: H.W.L., J.W.J., E.J.J., and C.H.L. Manuscript drafting: H.W.L., E.J.J., and C.H.L. Critical revision of the manuscript and important intellectual content: E.J.J. and C.H.L. Study supervision: C.H.L. 


\section{References}

1 GBD 2017. Causes of Death Collaborators. Global, regional, and national age-sex-specific mortality for 282 causes of death in 195 countries and territories, 1980-2017: a systematic analysis for the Global burden of disease study 2017. Lancet. 2018;392:173688.

2 Donaldson GC, Wedzicha JA. COPD exacerbations .1: epidemiology. Thorax. 2006;61(2): 164-8.

3 Teo WS, Tan WS, Chong WF, Abisheganaden J, Lew YJ, Lim TK, et al. Economic burden of chronic obstructive pulmonary disease. Respirology. 2012;17(1):120-6.

4 Global Initiative for Chronic Obstructive Lung Disease. Global strategy for the diagnosis, management, and prevention of chronic obstructive pulmonary disease 2020 report. 2019. Available from: https://goldcopd.org/ wp-content/uploads/2019/12/GOLD2020-FINAL-ver1.2-03Dec19_WMV.pdf. Accessed 2019 December 30.

5 Calverley PM, Anderson JA, Celli B, Ferguson GT, Jenkins C, Jones PW, et al. Salmeterol and fluticasone propionate and survival in chronic obstructive pulmonary disease. $\mathrm{N}$ Engl $\mathrm{J}$ Med. 2007;356(8):775-89.

6 Oba Y, Keeney E, Ghatehorde N, Dias S. Dual combination therapy versus long-acting bronchodilators alone for chronic obstructive pulmonary disease (COPD): a systematic review and network meta-analysis. Cochrane Database Syst Rev. 2018;12:Cd012620.

7 Fuso L, Mores N, Valente S, Malerba M, Montuschi P. Long-acting beta-agonists and their association with inhaled corticosteroids in COPD. Curr Med Chem. 2013;20(12):147795.

8 Montuschi P, Macagno F, Valente S, Fuso L. Inhaled muscarinic acetylcholine receptor antagonists for treatment of COPD. Curr Med Chem. 2013;20(12):1464-76.

9 Lipson DA, Barnhart F, Brealey N, Brooks J, Criner GJ, Day NC, et al. Once-daily singleinhaler triple versus dual therapy in patients with COPD. N Engl J Med. 2018;378(18): 1671-80.

10 Vestbo J, Anderson JA, Brook RD, Calverley PM, Celli BR, Crim C, et al. Fluticasone furoate and vilanterol and survival in chronic obstructive pulmonary disease with heightened cardiovascular risk (SUMMIT): a double-blind randomised controlled trial. Lancet. 2016;387(10030):1817-26.

11 Montuschi P, Malerba M, Macis G, Mores N, Santini G. Triple inhaled therapy for chronic obstructive pulmonary disease. Drug Discov Today. 2016;21(11):1820-7.

12 Papi A, Vestbo J, Fabbri L, Corradi M, Prunier H, Cohuet G, et al. Extrafine inhaled triple therapy versus dual bronchodilator therapy in chronic obstructive pulmonary disease (TRIBUTE): a double-blind, parallel group, randomised controlled trial. Lancet. 2018; 391(10125):1076-84.
13 Vestbo J, Papi A, Corradi M, Blazhko V, Montagna I, Francisco C, et al. Single inhaler extrafine triple therapy versus long-acting muscarinic antagonist therapy for chronic obstructive pulmonary disease (TRINITY): a double-blind, parallel group, randomised controlled trial. Lancet. 2017;389(10082): 1919-29.

14 Calverley PMA, Anzueto AR, Carter K, Grönke L, Hallmann C, Jenkins C, et al. Tiotropium and olodaterol in the prevention of chronic obstructive pulmonary disease exacerbations (DYNAGITO): a double-blind, randomised, parallel-group, active-controlled trial. Lancet Respir Med. 2018;6(5):337-44.

15 Singh D, Papi A, Corradi M, Pavlisova I, Montagna I, Francisco C, et al. Single inhaler triple therapy versus inhaled corticosteroid plus long-acting beta2-agonist therapy for chronic obstructive pulmonary disease (TRILOGY): a double-blind, parallel group, randomised controlled trial. Lancet. 2016; 388:963-73.

16 Lipson DA, Barnacle H, Birk R, Brealey N, Locantore $\mathrm{N}$, Lomas DA, et al. FULFIL trial: once-daily triple therapy for patients with chronic obstructive pulmonary disease. Am J Respir Crit Care Med. 2017;196(4):438-46.

17 Scuri M, Vestbo J, Papi A, Corradi M, Montagna I, Francisco C, et al. P271 Effect of extrafine single inhaler triple therapy on lung function and use of rescue medication: results from the trinity study. BMJ Publishing Group Ltd; 2017.

18 Lee HW, Park J, Jo J, Jang EJ, Lee CH. Comparisons of exacerbations and mortality among regular inhaled therapies for patients with stable chronic obstructive pulmonary disease: systematic review and Bayesian network meta-analysis. PLoS Med. 2019;16(11): e1002958.

19 Frith PA, Thompson PJ, Ratnavadivel R, Chang CL, Bremner P, Day P, et al. Glycopyrronium once-daily significantly improves lung function and health status when combined with salmeterol/fluticasone in patients with COPD: the GLISTEN study, a randomised controlled trial. Thorax. 2015;70(6):519-27.

20 Tricco AC, Strifler L, Veroniki AA, Yazdi F, Khan PA, Scott A, et al. Comparative safety and effectiveness of long-acting inhaled agents for treating chronic obstructive pulmonary disease: a systematic review and network metaanalysis. BMJ open. 2015;5(10):e009183.

21 Hutton B, Salanti G, Caldwell DM, Chaimani A, Schmid CH, Cameron C, et al. The PRISMA extension statement for reporting of systematic reviews incorporating network metaanalyses of health care interventions: checklist and explanations. Ann Intern Med. 2015; 162(11):777-84.

22 Spiegelhalter DJ, Myles JP, Jones DR, Abrams KR. Bayesian methods in health technology assessment: a review. Health Technol Assess. 2000;4(38):1-130.
23 McGowan J, Sampson M, Salzwedel DM, Cogo E, Foerster V, Lefebvre C. PRESS peer review of electronic search strategies: 2015 guideline statement. J Clin Epidemiol. 2016; 75:40-6.

24 Moher D, Liberati A, Tetzlaff J, Altman DG. Preferred reporting items for systematic reviews and meta-analyses: the PRISMA statement. PLoS Med. 2009;6(7):e1000097.

25 Liu Z, Rich B, Hanley JA. Recovering the raw data behind a non-parametric survival curve. Syst Rev. 2014;3:151.

26 Mackay AJ, Donaldson GC, Patel AR, Singh R, Kowlessar B, Wedzicha JA. Detection and severity grading of COPD exacerbations using the exacerbations of chronic pulmonary disease tool (EXACT). Eur Respir J. 2014; 43(3):735-44.

27 Burge S, Wedzicha JA. COPD exacerbations: definitions and classifications. Eur Respir J Suppl. 2003;41:46s-53s.

28 Higgins JP, Altman DG, Gøtzsche PC, Jüni P, Moher D, Oxman AD, et al. The cochrane collaboration's tool for assessing risk of bias in randomised trials. BMJ. 2011;343:d5928.

$29 \mathrm{Lu} \mathrm{G}$, Ades AE. Combination of direct and indirect evidence in mixed treatment comparisons. Stat Med. 2004;23(20):3105-24.

30 Dias S, Welton NJ, Sutton AJ, Ades AE. NICE decision support unit technical support documents; NICE DSU technical support document 2: a generalised linear modelling framework for pairwise and network meta-analysis of randomised controlled trials. London: $\mathrm{Na}$ tional Institute for Health and Care Excellence (NICE); 2014.

31 Carlin BP, Hong H, Shamliyan TA, Sainfort F, Kane RL. Case study comparing bayesian and frequentist approaches for multiple treatment comparisons; 2013.

32 Inhaled corticosteroids: their role in chronic obstructive pulmonary disease. MeReC Bull 2000;11:21-4.

33 Dias S, Welton NJ, Caldwell DM, Ades AE. Checking consistency in mixed treatment comparison meta-analysis. Stat Med. 2010; 29(7-8):932-44.

34 EU Clinical Trials Register. A multinational, multicentre, randomised, open-label, active-controlled, 26-week, 2-arm, parallel group study to evaluate the non-inferiority of fixed combination of beclometasone dipropionate plus formoterol fumarate plus glycopyrronium bromide administered via pMDI (CHF 5993) versus fixed combination of fluticasone furoate plus vilanterol administered via DPI (Relvar ${ }^{ø}$ ) plus tiotropium bromide $\left(\right.$ Spiriva $\left.^{\circ}\right)$ for the treatment of patients with chronic obstructive pulmonary disease. Available at: https://www.clinicaltrialsregister. $\mathrm{eu} / \mathrm{ctr}$-search/trial/2014-001487-35/results. Accessed 2020 Feb 19.

35 Zheng Y, Zhu J, Liu Y, Lai W, Lin C, Qiu K, et al. Triple therapy in the management of chronic obstructive pulmonary disease: systematic review and meta-analysis. BMJ. 2018;363:k4388. 
36 Cazzola M, Rogliani P, Calzetta L, Matera MG. Triple therapy versus single and dual long-acting bronchodilator therapy in COPD: a systematic review and meta-analysis. Eur Respir J. 2018;52:1801586.

37 Calzetta L, Cazzola M, Matera MG, Rogliani P. Adding a LAMA to ICS/LABA Therapy: a meta-analysis of triple combination therapy in COPD. Chest. 2019;155(4):758-70.

38 Lai CC, Chen $\mathrm{CH}$, Lin CYH, Wang CY, Wang $\mathrm{YH}$. The effects of single inhaler triple therapy vs single inhaler dual therapy or separate triple therapy for the management of chronic obstructive pulmonary disease: a systematic review and meta-analysis of randomized controlled trials. Int J Chron Obstruct Pulmon Dis. 2019;14: 1539-48.

39 Dulai PS, Singh S, Patel J, Soni M, Prokop LJ, Younossi Z, et al. Increased risk of mortality by fibrosis stage in nonalcoholic fatty liver disease: Systematic review and meta-analysis. Hepatology. 2017;65(5):1557-65.
40 Bremner PR, Birk R, Brealey N, Ismaila AS, Zhu C-Q, Lipson DA. Single-inhaler fluticasone furoate/umeclidinium/vilanterol versus fluticasone furoate/vilanterol plus umeclidinium using two inhalers for chronic obstructive pulmonary disease: a randomized non-inferiority study. Respir Res. 2018; 19(1):19.

41 Singh D, Corradi M, Spinola M, Papi A, Usmani OS, Scuri M, et al. Triple therapy in COPD: new evidence with the extrafine fixed combination of beclomethasone dipropionate, formoterol fumarate, and glycopyrronium bromide. Int J Chron Obstruct Pulmon Dis. 2017;12:2917-28.

42 Lee HW, Park J, Jang EJ, Lee C-H. Comparisons of exacerbations and mortality among LAMA/LABA combinations in stable chronic obstructive pulmonary disease: systematic review and Bayesian network meta-analysis. Respir Res. 2020 Nov;21(1): 310 .
43 Crim C, Calverley PM, Anderson JA, Celli B, Ferguson GT, Jenkins C, et al. Pneumonia risk in COPD patients receiving inhaled corticosteroids alone or in combination: TORCH study results. Eur Respir J. 2009; 34(3):641-7.

44 Allen A, Bareille PJ, Rousell VM. Fluticasone furoate, a novel inhaled corticosteroid, demonstrates prolonged lung absorption kinetics in man compared with inhaled fluticasone propionate. Clin Pharmacokinet. 2013;52(1): $37-42$.

45 Montuschi P, Malerba M, Santini G, Miravitlles M. Pharmacological treatment of chronic obstructive pulmonary disease: from evidencebased medicine to phenotyping. Drug Discov Today. 2014;19(12):1928-35.

46 Martinez FJ, Donohue JF, Rennard SI. The future of chronic obstructive pulmonary disease treatment--difficulties of and barriers to drug development. Lancet. 2011; 378(9795):1027-37. 